\title{
L'imposition de la norme européenne au Canada au dix-septième siècle à travers une perspective écocritique
}

\section{Eric D'AVERNAS}

Dans le cadre de l'approche écocritique, il faut voir les humains comme des êtres vivants ayant un rapport symbiotique, complexe et dynamique avec l'environnement qui les entoure. Cette appartenance humaine à une entité beaucoup plus vaste, mais toujours vivante est la raison pour laquelle nos actions, volontaires ou involontaires, affectent directement le présent et l'avenir. Dans Ecology without Nature, Timothy Morton illustre bien cette pensée écologique en soulignant que le sens familier de «nature » doit changer si nous voulons améliorer notre façon de vivre: «Ecology without Nature argues that the very idea of 'nature' which so many hold dear will have to wither away in an 'ecological' state of human society. Strange as it may sound, the idea of nature is getting in the way of properly ecological forms of culture, philosophy, politics, and art » (1). Pour Morton, donc, la notion même de nature pose des problèmes; il faut redéfinir la «nature » et ce qui est «naturel» afin de vivre de manière plus écologique. Autrement dit, si nous voulons exister de façon viable, durable et en harmonie avec le monde naturel, il faut laisser tomber l'idée qu'il existe une nature «non humaine »; bien au contraire, les humains font autant partie de la nature que le reste des espèces de la planète.

La relation de voyage est un genre littéraire très intéressant dans le cadre d'une étude sur l'environnement et cela pour plusieurs raisons. Pendant la période des Grandes découvertes, beaucoup de voyageurs européens sont arrivés au Nouveau Monde captivés par les singularités et déterminés à relater ce qu'ils voyaient. Bien entendu, on découvrait une nouvelle flore, une faune inconnue, des terres et des rivières, mais l'Amérique était aussi une terre de rencontres. Les différences entre ici et là nécessitaient des descriptions écrites pour que les explorateurs puissent rapporter la connaissance à leurs pays natals. La rencontre des explorateurs européens et des autochtones du Canada nous offre une occasion particulière pour réfléchir à la dichotomie entre les espaces naturels et l'œuvre humaine d'aujourd'hui. Les descriptions de la nature et des autochtones dans les Voyages de Samuel de Champlain, par exemple, nous montrent que la fondation de la Nouvelle-France et l'imposition de la culture européenne en Amérique étaient le début d'une nouvelle norme ayant repoussé et remplacé celle des indigènes. Les récits de voyage 
décrivant la colonisation sont ainsi une fenêtre par laquelle nous pouvons voir comment une culture qui fonde une nouvelle colonie permanente se frotte à la culture originelle qui y demeure depuis très longtemps. Ainsi, une analyse écocritique des textes anciens comme ceux de Champlain est nécessaire pour comprendre le monde dans lequel nous vivons aujourd'hui, surtout par rapport aux normes établies il y a des centaines d'années. Avec la colonisation française du Canada au dix-septième siècle, les autochtones commencent à occuper un espace intermédiaire entre le nouvel espace européen et la nature. L'objectif français d'imposer la culture européenne et la religion catholique force les autochtones à s'éloigner de leur ancien rapport à l'environnement, et la domination européenne s'affirmant, ils jouent dès lors un rôle secondaire dans l'entreprise de la Nouvelle-France.

Dans cet article, j'analyse les Voyages de Samuel de Champlain et la façon dont la fondation de la Nouvelle-France écarte la norme autochtone. Cette étude s'effectue en trois temps. Je montre d'abord le rapport de Champlain et de ses compatriotes à la nature, pour ensuite souligner comment la fondation des habitations européennes nuit à la norme autochtone. Finalement, j'analyse deux illustrations que Champlain a dessinées et qui montrent une autre manière dont la marginalisation des autochtones par rapport aux Européens au Canada se manifeste dans son œuvre. Cette communication montre également comment la nature, à laquelle les autochtones sont étroitement liés selon la perspective de Champlain, se marginalise de la même manière.

\section{Écocritique}

L'écocritique est l'étude du rapport entre l'humain et la nature. Même si les spécialistes de l'écocritique explorent des thèmes de la nature, ce n'est pas uniquement une étude thématique parce qu'ils mettent en question le sens même du mot «Nature ». Par exemple, on a tendance aujourd'hui à imaginer que la nature se trouve à l'extérieur des grandes concentrations humaines. La campagne, la forêt, la montagne, la mer et l'étendue sauvage se trouvent à l'extérieur des milieux urbains. Pour y accéder, il faut quitter la ville. Plus l'endroit naturel est loin des villes, moins il attire l'être humain. La «nature non humaine » est dans ce sens plus pure, dénuée de l'altération humaine, et fait partie de notre imaginaire d'un monde naturel vierge et lointain. La «nature humaine », par comparaison, fait référence aux endroits où les humains habitent et qu'ils visitent fréquemment. L'écocritique suggère que la «nature non humaine » n'existe pas réellement, ou n'existe qu'à peine, parce que les effets de l'activité humaine résonnent 
mondialement, surtout en raison de la globalisation. Comprendre ainsi le rapport intime entre les humains et le monde nous permet d'agir de façon appropriée et efficace afin d'empêcher et, éventuellement, d'arrêter les dommages environnementaux. C'est ce type de changements réels dans le monde que l'écocritique vise à effectuer. Le spécialiste de l'écocritique veut non seulement analyser, mais aussi appliquer ses recherches ou ses perspectives à la conscience environnementale actuelle ${ }^{1}$.

Les Européens du dix-septième siècle voyaient la nature d'une manière très différente de celle des autochtones. Considérons maintenant ce qu'était la nature à l'époque pour les colonisateurs.

\section{La perspective européenne envers la nature et les autochtones}

Les Européens de l'époque à l'étude se sont déjà éloignés des forêts (autrement dit «la nature ») pour vivre dans des centres de culture humaine (urbains ou semi-urbains), où ils manipulent la nature à leurs fins. Les autochtones, par contre, peuples nomades ou semisédentaires, comptaient sur la forêt qui les entourait pour survivre.

Comme l'habitation est le centre de la présence européenne sur la terre ferme, il est important de considérer comment Champlain représentait les villes. Sa perception du rapport entre les villes et la nature ressort notamment dans une description de la ville de Gênes dans l'édition de 1632 de ses Voyages : «Gênes, l'une des plus superbes villes du monde, édifiée dans un pays environné de montagnes, fort désert et si infertile que les habitants sont contraints de faire apporter la terre de dehors pour cultiver leurs jardins d'alentour [...]» (Au secours de l'Amérique française, 42). Le jardin représente une forme de nature contrôlée par les humains qui se trouve à l'intérieur de la ville. La terre vient de l'extérieur, d'un autre espace, mais les humains doivent la travailler afin de la rendre fertile. En transférant cette façon de vivre et de construire des villes de l'Europe à la Nouvelle-France, la présence européenne au Canada menace et transforme l'équilibre du rapport (qui y était déjà établi) entre l'humain et la nature.

Champlain propose ensuite une description semblable de Marseille qui renforce cette image de la nature contrôlée par l'homme, l'auteur écrit ainsi : «La ville de Marseille, qui autrefois n'était qu'un marécage, environné de collines et de montagnes assez fâcheuses, néanmoins par succession de temps a rendu son territoire fertile et est devenue fameuse et grandement marchande. Ainsi plusieurs petites colonies ayant la commodité des ports et des 
havres, se sont accrues en richesses et réputations » (Au secours de l'Amérique française, 42). Champlain souligne ainsi la présence indésirable et fâcheuse du marais qui disparaît après les changements entrepris par la ville de Marseille. Le fait qu'elle devienne «fameuse » et « grandement marchande » renforce l'importance du commerce du point de vue des Européens. Le lien qu'il fait entre la réputation positive, l'accumulation de richesses et le développement de la ville, souligne également l'intérêt du voyageur pour le commerce. De plus, cet exemple sert à Champlain à montrer que même des terres stériles peuvent se transformer en une ville prospère, pourvu que l'accès à l'eau soit sûr et fiable, ce qui renforce aussi la dépendance aux cours d'eau et à la mer. Comme il le souligne, les nombreuses colonies qui « se sont accrues en richesses et réputation » grâce en partie aux ports et aux havres commodes, font souvent allusion aux sites considérés pour l'établissement des habitations. Il laisse ainsi entendre que le développement des colonies en Nouvelle-France peut se faire facilement, comme on l'avait déjà fait en Europe avec les villes.

Un emploi rhétorique ressort également de la description de Marseille de Champlain : il personnifie la ville. Selon le passage, c'est la ville elle-même qui « a rendu son territoire fertile » et qui œuvre afin d'altérer le paysage qui l'entoure. Avant cette transformation, elle «n'était qu'un marécage », un lieu naturel. Ainsi, l'action humaine transforme le lieu en ville qui se développe par la suite; la ville est à la fois un espace physique et un être vivant qui vit et évolue en même temps que les humains qui l'habitent. La ville s'émancipe à la fois des mains de l'humain et de la nature; elle ne fait plus partie des collines et des montagnes qui l'environnent. La vie humaine (pour les Européens) est donc au centre de la ville et l'anime. Telle est la vision des villes selon Champlain. Il encourage leur développement au Canada en perpétuant la séparation entre la ville et la nature, selon la vision sociale européenne. Par contre, les autochtones, dans les Voyages de Champlain, ne font pas la même distinction entre la nature et la demeure humaine.

La terre représente donc, pour Champlain, un lieu en devenir, prêt à accueillir la ville européenne. Son entreprise en Nouvelle-France a pour objectif d'effectuer cette implantation permanente. Tandis que les habitations autochtones se trouvent au cœur des forêts, celles des Européens s'éloignent de la nature. De sa perspective, le pays est un jardin rempli de ressources que les Français doivent entretenir. Les jardins à l'intérieur des villes, plantés et arrangés géométriquement de la main des français, représentent d'autant plus la manipulation de la nature. 
Champlain souligne néanmoins que la beauté de la terre dépend de l'eau qui la rend accessible aux navires français, toujours dans l'objectif de trouver des endroits pour établir des habitations. Pourvu qu'une voie navigable permette aux Français de débarquer sans encombre en NouvelleFrance, les colonisateurs ont le pouvoir, s'ils le veulent bien, de prendre le contrôle d'un espace et de le transformer afin de le rendre utile à leurs besoins. Ce faisant, l'espace autochtone au Canada cède la place au nouvel espace européen au fil du temps.

En ce qui concerne la vision que Champlain a des autochtones, il les place explicitement au sein de la forêt. Le terme "sauvage », que Champlain emploie constamment dans ses Voyages, a une étymologie intéressante par rapport aux autochtones qui, selon Champlain, «vivent dans la forêt ». En effet, le terme a pour étymologie le mot latin «silva» ou «forêt», l'emploi du mot « sauvage » a une signification importante à l'époque de Champlain. Que ce soit un adjectif ou un substantif, "sauvage » veut dire être physiquement séparé de la civilisation que les Européens connaissaient depuis longtemps. Autrement dit, la forêt (et les autres lieux naturels inhabités) et les autochtones qui y vivent, représentent fondamentalement, pour Champlain, le «non-civilisé ». Les habitations européennes au Canada se démarquent de la demeure sylvestre des autochtones au moment de leur établissement. Ainsi, la fondation de la Nouvelle-France crée un espace européen, « civilisé » qui s'agrandit au fur et à mesure.

Puisque Champlain considérait les autochtones comme des humains non civilisés faisant partie de la forêt, ses descriptions nous permettent non seulement de voir comment les colonisateurs ont imposé leur norme au Canada, mais aussi de comprendre, selon la perspective européenne, comment les autochtones et l'espace qu'ils occupaient (autrement dit la nature) sont devenus marginalisés. La colonisation du Canada crée un nouvel espace, uniquement européen, qui se frotte à celui des autochtones et le remplace avec le temps.

\section{Iconographie}

Une autre caractéristique intéressante du genre de la relation de voyage est la présence de l'iconographie. Dans un premier temps, les gravures ajoutent une dimension visuelle complémentaire aux descriptions de l'autre. Les auteurs essayaient de faire une esquisse de ce qu'ils voyaient afin de renforcer leurs descriptions. Dans un deuxième temps, en rassemblant toutes les gravures d'un explorateur, nous pouvons mettre en relief les évolutions présentes, tout comme le fait Frank Lestringant pour André Thevet ${ }^{2}$. S'il est vrai que «Pour l'historien, les mots 
sont plus fiables que les images » (François-Marc Gagnon 307) et qu'il faut donc se méfier de la vérité des illustrations, les images possèdent tout de même une certaine valeur. De la même manière que les mots d'un récit peuvent cacher d'autres significations ou préjugés, les illustrations ne montrent pas forcément l'exacte réalité. En analysant les images en même temps que les descriptions, nous arriverons à une compréhension plus complète de la perspective de l'époque. L'iconographie dans la relation de voyage révèle ainsi autant d'information sur le sujet dessiné que sur l'auteur lui-même.

Les dessins de Champlain nous permettent d'analyser une autre dimension du monde de la Nouvelle-France. Chaque gravure est un portrait d'un ou de plusieurs endroits vus par Champlain. Qu'il s'agisse de son écriture ou de ses dessins, Champlain choisit un sujet précis. Il faut donc garder à l'esprit qu'il ne s'agit pas nécessairement d'une représentation parfaite de l'espace ni d'un instantané. Les gravures montrent un idéal afin de faire voir aux lecteurs ce qu'il considère être digne d'une représentation. Il dessine parfois des scènes très dynamiques (telles que les batailles et les cérémonies autochtones) et essaye de tout montrer dans un seul portrait. Comme l'ensemble de son œuvre, ses représentations sont ainsi un résumé de ce qui se passe et de ce qu'il voit au Canada. Même si elles sont peut-être «en défaut par rapport à la « réalité » » (Gagnon 304) ou que le graveur insère des «erreurs ethnographiques » (307), c'est-à-dire des ajouts qui n'appartiennent pas à Champlain, il faut tenir compte de la valeur qui réside dans ce que Champlain voit et ce qu'il veut montrer.

À travers ses Voyages, Champlain produit de nombreuses illustrations dépeignant des sujets variés qui peuvent être classés en trois catégories principales : les cartes, les habitations et les autochtones. Dans le cadre de cet article traitant surtout du rapport entre les Européens et la nature, examinons une gravure de l'habitation de l'île de Sainte-Croix, fondée par le Sieur Dugua de Mons en 1604 : 
Figure 1. « Habitation de l'Ile Ste-Croix »

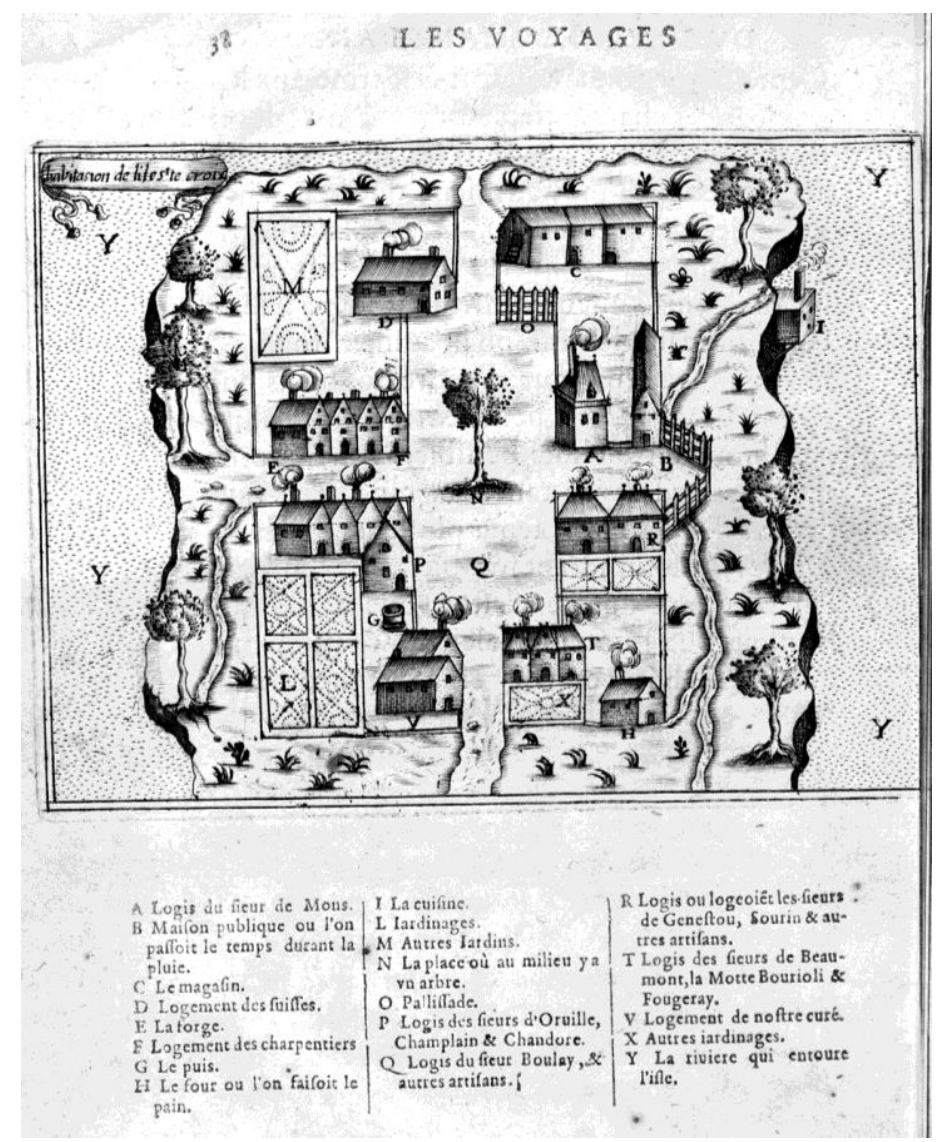

D'abord, il faut noter la forme rectangulaire de l'habitation. Tous les jardins et les bâtiments, sauf la cuisine qui se situe près du fleuve et fait usage de l'eau courante, se trouvent à l'intérieur de limites définies, c'est-à-dire des palissades qui entourent les constructions. Hormis les quelques jardins, la seule plante visible à l'intérieur de l'habitation est un arbre entièrement centré. À l'extérieur se trouve de l'herbe qui pousse partout. Ce contraste suggère qu'à l'intérieur de l'habitation les colonisateurs ont défriché la terre pour la nettoyer. Ils créent ainsi un nouvel espace sculpté et séparé de la terre non cultivée de l'extérieur. Comme nous l'avons vu dans les descriptions de Gênes et de Marseille, la construction de cette habitation repousse effectivement la nature hors de la demeure humaine. 
Les nombreux jardins de l'habitation_symbolisent l'enfermement des plantes qui poussent dans un espace clos, contrôlé par les humains. Même si les plantes sont d'origine canadienne, ce sont les Européens qui gèrent leur croissance. De plus, les colonisateurs ont planté chacun des jardins dans la gravure de manière géométrique, « une conception raffinée, rappelant les jardins classiques de France » (Fischer 287). L'importation de ce modèle européen est surprenante dans un pays aussi vaste, mais laisse entendre que les Européens ont besoin de contrôler la nature. La distinction entre l'espace habité et les espaces naturels hors de l'habitation est bien marquée : l'homme européen ne peut ainsi faire partie intégrante de la nature.

Cependant, l'arbre au centre de l'habitation s'oppose à cette séparation et peut suggérer que la nature «non humaine» se trouve toujours à l'intérieur des palissades; pourtant, sa position n'est pas sans signification. Au-dessous de la gravure, Champlain note que le centre est «La place où au milieu y a vn arbre ». Si nous reprenons la seule définition du mot «place » du Dictionnaire Godefroy, définition qui est très générale, une place est un «lieu assigné à une personne, à une chose, à un usage » (346). L'adjectif « assigné » signifie qu'un humain décide de la valeur du lieu, et cela montre que l'arbre est utile pour les habitants, sans aucun doute comme point de rendez-vous ou de référence. Selon le Dictionnaire électronique de Chrétien de Troyes, une «place» dans le sens que Champlain emploie, sans doute, est plus précisément un «[e] Espace découvert, lieu public» («Place », 1), où l'on plantait souvent un «arbre de mai » (Gay 102). L'emplacement de l'arbre a sans doute un aspect esthétique, mais cela a également une valeur humaine. De plus, du fait que six autres arbres semblables se trouvent à la périphérie de l'île, on peut supposer que les colonisateurs en ont coupé plusieurs autres pour faire de la place pour les bâtiments et les jardins. Celui du centre reste debout toujours par hasard parce qu'ils ont décidé d'y laisser un espace ouvert pour le carrefour.

La perspective de cette gravure est une autre caractéristique intéressante. Toute l'île est représentée sur un seul plan et il semble qu'elle suive la forme rectangulaire de l'habitation. La carte géographique à la page précédente dans l'édition Thierry montre que l'île n'a pas les mêmes angles droits et nous pouvons voir que l'île, aujourd'hui, n'a pas cette forme ${ }^{3}$ (Champlain, Fondations de l'Acadie et de Québec, 74). De plus, il n'est pas facile d'identifier la nature des limites de l'île, entre falaises ou plages alignées avec la rivière. Il réduit ainsi les caractéristiques physiques de l'île au plan qui représente le mieux l'habitation, déformant effectivement la géographie du lieu. Même s'il est difficile ou impossible de montrer la 
topographie, l'essentiel est que Champlain essaye de montrer dans cette esquisse la demeure européenne et non pas la nature.

Le fait que Champlain choisisse l'habitation comme sujet de la gravure est également important. En procédant ainsi, Champlain montre qu'il accorde une certaine importance à l'habitation et à son organisation. D'autre part, il pense probablement que cet aspect de l'entreprise en Nouvelle-France intéressera les lecteurs de son œuvre. Il avait sans aucun doute raison, car ses lecteurs reconnaîtront les caractéristiques européennes de l'habitation et comprendront comment lui et ses compatriotes parviennent à vivre au Canada. La gravure représente l'ensemble de la résidence européenne, mais en la dessinant, Champlain fait ressortir sa propre perspective sur la place de la nature à l'époque.

La gravure de l'île de Sainte-Croix confirme que les habitations au Canada suivent le modèle de la ville européenne selon Champlain. Elle renforce le fait que les colonisateurs manipulent la nature et s'en éloignent dès leur première tentative d'implantation. Les jardins, à l'intérieur de l'habitation, cultivés de manière européenne, et le défrichement de la terre affirment également le contrôle qu'ils exercent sur l'environnement qui les entoure afin de le rendre «familier $»^{4}$. En construisant l'habitation de cette façon, Champlain et les colonisateurs ouvrent la voie au développement des grandes villes européennes qui mettent l'accent sur l'efficacité et mettent à l'écart la nature, illustrant bien le rapport entre les Européens et leur environnement.

La prochaine illustration que nous allons examiner s'intitule la « Défaite des Iroquois au lac Champlain ». Elle dépeint une scène très dynamique, une bataille où l'alliance de trois nations autochtones différentes (avec trois hommes français) affronte les Iroquois : 


\section{Figure 2. « Défaite des Iroquois au lac Champlain »}

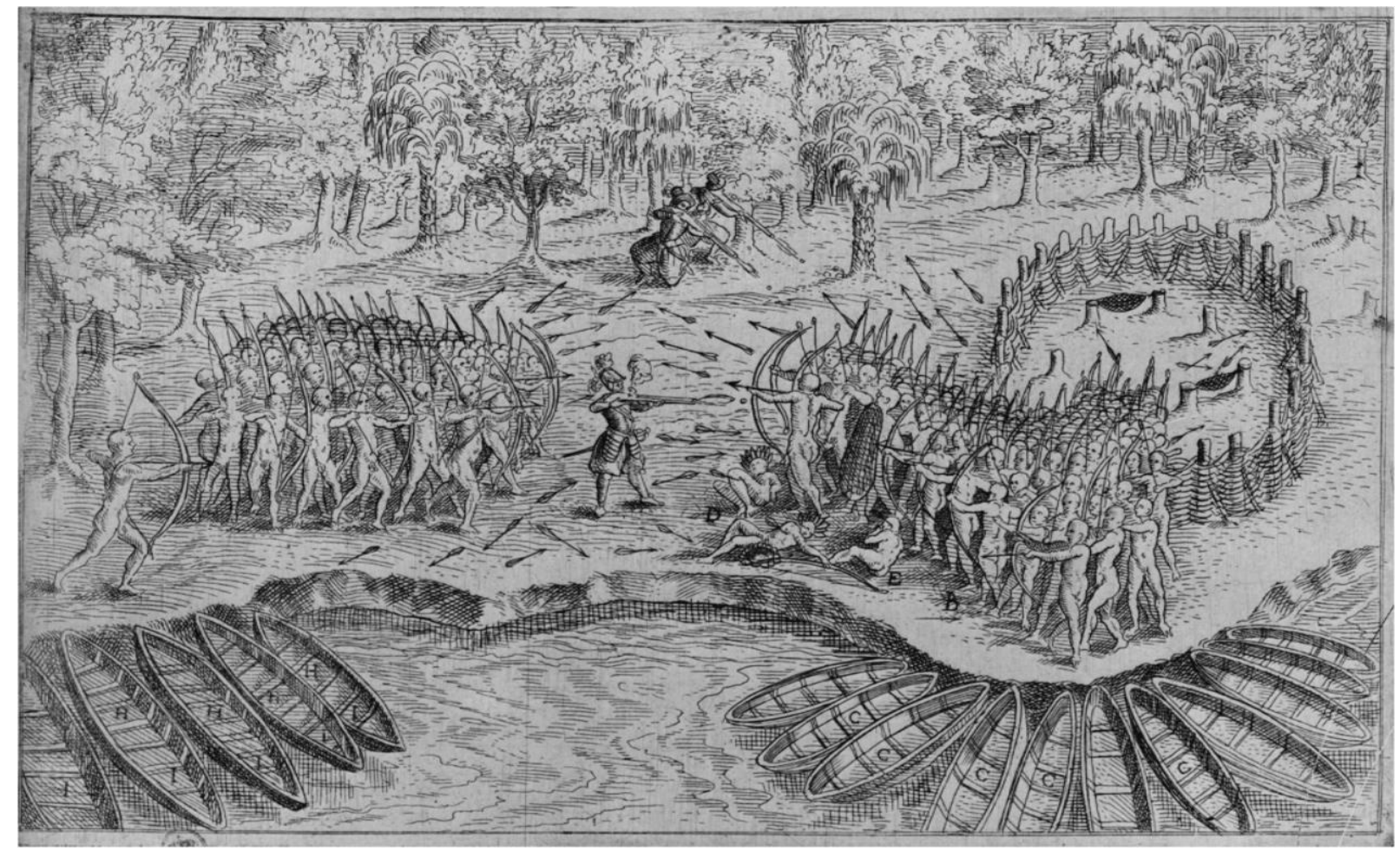

Commençons par décrire ce que Champlain a tenté de représenter dans cette illustration. Au plan intermédiaire de cette scène se trouvent les deux groupes d'autochtones se faisant face et tirant à l'arc, et Champlain accompagné de deux hommes tirant sur les Iroquois avec des arquebuses instrument dont l'ingénierie dénote d'ores et déjà la domination européenne. D'après la description de cette bataille, les compagnons de Champlain tirent des coups de fusil de l'orée de la forêt vers l'espace au centre de l'illustration, là où se déroule l'action principale. Les représentations variées des différents peuples attirent l'œil. Les autochtones n'ont pas de caractéristiques physiques qui les distinguent les uns des autres, sauf les deux chefs à terre portant des coiffures ; un seul Iroquois manie un bouclier. Par conséquent, les deux troupes sont réduites à des masses indiscernables d'autochtones qui se mélangent dans la foule. La description qui accompagne cette illustration, confirme cette image des autochtones puisque Champlain nous dit : «Je vis sortir les ennemis de leur barricade, qui étaient près de 200 hommes forts et robustes à voir, qui venaient au petit pas au-devant de nous, avec une gravité et une assurance qui me contentèrent fort, à la teste desquels il y avait trois chefs » (Fondations de l'Acadie et de Québec, 200-201). La foule iroquoise qu'il dessine, représente la bande de deux cents guerriers qui se presse devant lui. Les trois Français, par contre, se démarquent et sont équipés par rapport à la 
nudité des autochtones, soulignant l'opposition entre les «civilisés » et les «non-civilisés ». Champlain lui-même se trouve seul au centre de la mêlée comme un brave guerrier. Certes, bien dépeindre plus de deux cents hommes sur une feuille de papier serait difficile, mais cette illustration souligne quand même l'importance qu'il accorde aux Européens et la vague perception qu'il a des groupes autochtones composés d'anonymes.

Le lieu de la bataille constitue un autre aspect important. Cette dernière se produit à la lisière de la forêt et au bord d'une étendue d'eau, dans une clairière sans doute partiellement déboisée par les Iroquois. L'image de l'espace intermédiaire entre l'eau et la forêt ressort dans cette illustration, comme dans les habitations européennes. Elle montre aussi l'importance des cours d'eau pour les déplacements au Canada, car toutes les nations y ont leurs canots arrimés et accessibles. À l'arrière-plan se trouve le bois épais d'où Champlain et ses alliés autochtones sont venus et dans lequel les Iroquois vaincus s'enfuient. Un parallèle intéressant ressort en comparant la représentation des arbres et celle des combattants : les arbres au premier plan sont comme les troupes d'autochtones les plus détaillés et dissimulent ceux plus à l'arrière dont on ne discerne que les troncs et les cimes, mais dont on imagine la multitude. Même si l'on ne voit que l'orée de la forêt, les rangs des arbres à l'arrière-plan laissent présager l'immensité de la forêt. La description de la fuite des Iroquois confirme l'étendue de la forêt telle que Champlain l'a dessinée. Curieusement, trois des arbres ressemblent à des palmiers avec des écorces écaillées. Ils ne sont pas originaires du Canada et représentent l'exotisme lié à l'Amérique en se démarquant des autres arbres à troncs lisses. Un autre parallèle s'établit également entre les trois arbres décalés et les trois Français, colonisateurs explorant un pays étranger puisque l'armure des Français attire l'attention notamment grâce au cuissard écaillé et à la plume sortant du casque.

Cette illustration accentue le portrait que Champlain fait des autochtones et de leur présence, au commencement de l'établissement de la Nouvelle-France et au cœur même de la nature. La représentation artistique va de pair avec les descriptions textuelles et permet aux lecteurs de distinguer aisément des autochtones. D'autre part, elle rend les Voyages plus crédibles, surtout grâce à cette dimension visuelle.

Les descriptions de Champlain nous montrent que les peuples indigènes sont intéressants et importants pour l'explorateur, en dépit de leur manque de civilisation évoqué tout au long des récits. Étant donné que l'écocritique étudie le rapport entre l'humain et la nature, il est utile de considérer la nature de la perspective autochtone. Pour ce faire, cependant, nous comptons 
sur les descriptions de Champlain, un Européen, qui a son propre point de vue en ce qui concerne les peuples autochtones et la nature. Cela entraîne un rapport tripartite entre les Européens qui créent des espaces humains en contrôlant les espaces naturels, la nature qui est étendue et qui se trouve ailleurs, et les autochtones qui occupent un espace quelque part entre les deux, plutôt dans la nature, mais qui interagissent avec les Européens.

Le concept qui joue le rôle le plus important dans les descriptions des peuples indigènes du Canada, est la dichotomie entre la culture humaine et les espaces naturels. Nous avons vu que les colonisateurs établissent un nouvel espace européen au Canada superposé à l'espace autochtone qui nuit à leur équilibre et à leur environnement. Étant donné que Champlain croit que la civilisation équivaut à la vie européenne et qu'il dit que certains autochtones ne font, dans ses mots, «aucune culture» (À la rencontre des Algonquins et des Hurons, 153), cette dichotomie implique plutôt le rapport européen à la nature. Par contre, d'un point de vue objectif, il semble que les autochtones ne distinguent pas leur demeure de la nature. Les Voyages de Champlain nous montrent qu'il existe d'autres manières de concevoir le monde, plus convenables à une pensée environnementale. À son époque, le Canada est un univers utopique, mi-européen et mi-autochtone. L'entreprise de la Nouvelle-France y donne accès aux Français. Il s'agit donc du commencement de l'essor de la norme européenne au Canada.

Comme toute étude écocritique doit (ou devrait) le faire, je termine avec des considérations actuelles. Nous vivons aujourd'hui dans une société qui provient de la colonisation européenne de l'Amérique du Nord, où « la vraie nature » se trouve en grande partie loin de notre demeure; la nature, cependant, est omniprésente, toujours dans un rapport symbiotique avec tout être vivant. De plus, la pensée environnementale (au Canada, par exemple) devrait tenir compte des peuples autochtones. Si l'on veut protéger l'environnement canadien, voire mondial, il ne faut pas oublier que nous, comme les autochtones de l'époque, en faisons partie. En soulignant l'espace que créent et occupent les Européens, nous sommes capables de repenser ainsi le rapport entre les humains et leur environnement. De la même manière, le portrait de l'Amérindien au dix-septième siècle nous aide à concevoir un autre monde avant la séparation évidente de la ville française et la propagation de la perspective européenne. D’ici la nécessité d'appliquer la théorie de l'écocritique aux textes anciens et fondateurs, qui ne se faisait pas, d'ailleurs, jusqu'à très récemment. Un tel constat est nécessaire pour un développement durable. 


\section{Bibliographie}

Champlain, Samuel de. Au secours de l'Amérique française. Dir. Éric Thierry. Québec: Septentrion, 2011.

---. À la rencontre des Algonquins et des Hurons. Dir. Éric Thierry. Québec : Septentrion, 2009.

---. Les Fondations de l'Acadie et de Québec. Dir. Éric Thierry. Québec : Septentrion, 2008.

---. «Habitation de 1'Ile Ste-Croix » [Illustrations de Les Voyages de Champlain]. Paris : J.

Berjon, 1613. En ligne : http://catalogue.bnf.fr/ark:/12148/cb38495058j

---. «Défaite des Iroquois au lac Champlain ». [Illustrations de Les Voyages de Champlain],

Paris : J. Berjon, 1613. http://catalogue.bnf.fr/ark:/12148/cb38495058j

Fischer, David Hackett. Le rêve de Champlain. Tr. Daniel Poliquin. Montréal : Boréal, 2011.

Gay, Victor. Glossaire archéologique du Moyen Age et de la Renaissance, t. 2. Paris : Société Bibliographique, 1887.

Godefroy, Frédéric-Eugène. Dictionnaire de l'ancienne langue française du IX siècle au $X V^{e}$ Siècle, t. 10. Genève: Slatkine, 1902. http://micmap.org/dicfro/search/dictionnairegodefroy

Joukovsky, Françoise. Paysages de la Renaissance. Paris : PUF, 1974.

Lestringant, Frank. «Les représentations du Sauvage dans l'iconographie relative aux ouvrages du cosmographe André Thevet». Bibliothèque d'humanisme et Renaissance, t. 40. Genève : Droz, 1978. 583-595. http://www.jstor.org/stable/20675942

Litalien, Raymonde et Denis Vaugeois, dir. Champlain. La naissance de l'Amérique française. Sillery et Paris : Septentrion et Nouveau Monde, 2004.

Morton, Timothy. Ecology without Nature: Rethinking Environmental Aesthetics. Cambridge, Mass. : Harvard UP, 2007.

«Place ». DÉCT: Dictionnaire Électronique de Chrétien de Troyes. Décembre 2014. LFA/Université d'Ottawa. 20 avril 2016 http://www.atilf.fr/dect

\footnotetext{
Notes

${ }^{1}$ Au sujet de l'écocritique, voir par exemple Greg Garrard, Ecocriticism, Londres et New York, Routledge, 2004 et Timothy Morton, The Ecological Thought, Cambridge (Massachusetts), Harvard UP, 2010.

2 Voir Frank Lestringant, "Les représentations du sauvage dans l'iconographie relative aux ouvrages du cosmographe André Thevet», Bibliothèque d'Humanisme et Renaissance, 1978, tome 40, p.583-595. http://www.jstor.org/stable/20675942.

3 Pour une image aérienne récente de l'Île Sainte-Croix, voir ce lien de l'Université du Maine : https://umaine.edu/canam/files/2009/10/st-croix-photov2-copy.jpg.

${ }_{4}^{4}$ J'évoque ici de nouveau le jardin comme «un décor familier» selon Françoise Joukovsky, Paysages de la Renaissance, p. 24.
} 